\title{
Prácticas contables y tributarias que adoptan los pequeños negocios en Colombia*
}

\section{Accounting and tax practices adopted by small businesses in Colombia}

\author{
Mario Heimer Flórez Guzmán \\ Ruby Stella Cabrera Jaramillo ${ }^{* * *}$ \\ Carlos Mario Londoño Toro ${ }^{* * * *}$
}

Fecha de recepción: 5 de julio de 2018

Fecha de aprobación: 20 de agosto de 2018

\section{RESUMEN}

El presente artículo tiene como objetivo describir las prácticas contables y tributarias de los pequeńos negocios en Colombia. Además, aborda el problema de que los negocios de barrio, por su estructura, tienden a relacionarse con el "sector informal", definido como el conjunto

* El presente ensayo es producto del proyecto de investigación "Incidencia de las medidas de formalización empresarial desde la perspectiva contable y tributaria en el desempeńo de los negocios barriales: el caso de la Comuna 10 de Medellín”, gestionado en la Corporación Universitaria Republicana. Citar como Flórez Guzmán, M., Cabrera Jaramillo, R. y Londońo Toro, C. (2019). Prácticas contables y tributarias que adoptan los pequeños negocios en Colombia. Via Inveniendi et Iudicandi, 14(1), 207-223. Doi: https:/doi. org/10.15332/s1909-0528.2019.0001.08

** Doctorado en Educación. Director del Centro de Investigaciones e Innovación en los Negocios. Corporación Universitaria Remington (Cali, Colombia). Orcid: https://orcid.org/0000-0002-8468-4168. mariohfg@hotmail.com

*** Magíster en Politica Fiscal y Tributo, Fundación Universitaria Luis Amigó. Docente de la Fundación Universitaria Luis Amigó. ruby.cabreraja@amigo.edu.co

**** Magister en Gestión de Organizaciones de la Universidad Cooperativa de Colombia. Docente de la Fundación Universitaria María Cano. carlosmariotrujillotorres@fumc.edu.co 
de actividades que se encuentran excluidas de la legislación o por fuera del ámbito impositivo. Asimismo, los negocios de barrio se entienden como aquellos establecimientos que realizan actividades económicas de pequeńa escala, en los cuales laboran trabajadores por cuenta propia y que en la mayoría de los casos contratan a familiares o amigos de manera informal. La investigación se soporta en la teoría de los stakeholders planteada por Freeman, según la cual las organizaciones establecen una relación biunívoca con las comunidades que las contienen: producen externalidades que afectan a estas últimas, mientras que las comunidades hacen que aquellas se configuren alrededor de sus necesidades. Metodológicamente la investigación es de corte positivista mixto con enfoque descriptivo y se ha utilizado el muestreo aleatorio simple como estrategia para la selección de la muestra. Después de analizada la información se llega a la conclusión de que las prácticas contables y tributarias formales en la población objeto de estudio son casi nulas, y que la causa de esta problemática es la falta de conocimiento en contabilidad, finanzas y tributación, así como los insuficientes mecanismos de control y apoyo de los organismos gubernamentales.

Palabras clave: prácticas contables, negocios barriales, mipyme. 


\begin{abstract}
This paper aims to describe accounting and tax practices of small companies in Colombia. In the other hand, describes the main matters faced by small business whose because of it's structure, tend to be linked with "informal market" taking into account that this sector has been defined as those who realize many activities legally exclude or out of the taxable field. In that order of ideas, grocery stores are known as business operating on a low scale, wich are integrated for employees working independently, also in most of cases, hire friends or family members in an informal way. From the theoretical component, research is supported by the Stakeholder theory proposed by Freeman, which establishes that organizations generate externalities to the communities in which they are immersed, from a biunivocal relationship, since communities also they make organizations configure themselves around their needs. From the methodological aspect, the research is mixed positivist with a descriptive approach and simple random sampling has been used as a strategy for the selection of the sample. After analyzing the information, it is concluded that formal accounting and tax practices in the population under study are almost nil, and the cause of this problem is the lack of knowledge in accounting, finance and taxation they have, as well as the insufficient mechanisms of control and support of government agencies to this population.
\end{abstract}

Keywords: Accounting practices, neighborhood businesses, MSMEs. 


\section{INTRODUCCIÓN}

La teoría jurídica es mucho más que idear recursos discursivos: es tal vez la tarea jurídica más exigente y compleja (Castro, 2018a). En este sentido, la normatividad colombiana en el marco contractual tendrá que verse reinterpretada (Castro, 2018b) desde la citada perspectiva. En Colombia estadísticamente se ha demostrado que los negocios barriales dinamizan gran parte del comercio y su influencia es directa en la fluctuación de las variables económicas por su participación como porcentaje del PIB. En este sentido, es procedente afirmar que desde finales del siglo XX el modelo clásico del derecho está viviendo un profundo agotamiento, debido a la insatisfacción de los modelos culturales y normativos (Hurtado, 2017, p. 19).

$\mathrm{Al}$ respecto, Llano señala:

Las transformaciones de la teoría del derecho de forma reciente, por factores como la amplia producción teórica en los Estados considerados centrales o la llegada de conceptos, interpretaciones de la realidad y corrientes teóricas de contextos académicos que fueron considerados marginales, determina un horizonte de deliberaciones teóricas que tienen la pretensión de comprender fenómenos contemporáneos donde las ciencias sociales, las especialidades de la ciencia jurídica y la misma teoría del derecho son esenciales. (2017, p. 31)

El VI Censo de Comercio, realizado por Servinformación, en su reporte final concluye que el $21 \%$ de los negocios en el país son tiendas de barrios, el $9 \%$ peluquerías y salones de belleza, el $8 \%$ restaurantes, seguidos por tiendas de ropa, cafés internet, droguerías y ferreterías. La realidad ubica a Colombia como un país de negocios de barrio (Dinero, 2016).

Este tipo de negocios engrosan la economía popular y solidaria, cuyo objetivo para quienes la integran es mejorar sus condiciones de ingresos monetarios de un modo que les permita alcanzar calidad de vida, no solo en función de la comercialización de bienes y servicios, sino de su propio consumo. 
La característica que identifica estos negocios es su domicilio en las zonas residenciales de los barrios de las grandes y pequeñas ciudades. La ubicación de algunos de ellos es la vía pública o espacios públicos donde han fijado su punto de venta. Igualmente, suelen operar en la informalidad: realizan su objeto social sin el pleno cumplimiento de las normas legales, laborales, contables y tributarias. Al imperar el conocimiento empírico empresarial, el análisis de la viabilidad y situación financiera del negocio se reduce al flujo de caja para identificar el margen de utilidad: ¿Cuánto gasto?; ¿cuánto vendo? La conclusión es lógica, el residuo de la operación matemática son las utilidades.

El presente artículo da cuenta de la cantidad y calidad de información contable y tributaria que manejan los pequeños negocios en la ciudad de Medellín. El documento se encuentra dividido en dos fases, una documental y la otra de corte experiencial en donde se expone la información recolectada mediante herramientas como encuestas y diarios de campo.

En concordancia con las líneas anteriores es importante resaltar que de acuerdo con el régimen de responsabilidad civil todo sujeto de derecho está en la obligación de responder por todos los perjuicios que cause en el cumplimiento de sus funciones a terceros de buena fe (Torres y Ramírez, 2018).

\section{Resultados Y DisCUSIONES}

\section{La formalidad y la informalidad en Colombia}

De conformidad con Herrera, "el hombre para vivir y sobrevivir ha introducido un conjunto amplio de leyes, convenciones, reglas y significados, los cuales otorgan unos elementos comunes que buscan la realización y el orden de nuestra sociedad" (2018, p. 58). Desde la citada perspectiva, la formalidad no puede concebirse únicamente como el registro ante la Cámara de Comercio, es el cumplimiento del ordenamiento jurídico en lo que respecta a las relaciones comerciales, laborales, administrativas y, ante todo, al vínculo jurídico que se desprende de la obligación tributaria al realizarse el hecho generador. 
El sector formal se asimila al informal en el momento en el que desconoce sus obligaciones legales, abusa de la norma laboral y tributaria a su favor o manipula la información financiera distorsionando la realidad económica. Es el caso de las empresas fantasma (Kanbur y Keen, 2014) que estando por encima del umbral tributario deciden declarar de forma fraudulenta, situándose por debajo del umbral para no declarar y no contribuir.

El Programa de Promoción de la Formalidad en América Latina y el Caribe liderado por la Organización Internacional del Trabajo considera la informalidad empresarial como

un fenómeno multidimensional donde intervienen diversos factores económicos, estructurales, institucionales y hasta políticos. También inciden fuertemente los procesos de globalización, externalización y subcontratación A nivel microeconómico, entre los principales determinantes que influyen en la decisión de las empresas de menor tamaño de operar informalmente se encuentran:

- La baja productividad de las micro y pequeñas empresas, que no permitiría enfrentar los costos de la formalidad.

- La complejidad de procedimientos para el registro de empresas y pago de obligaciones, así como la regulación inadecuada para las características de las micro y pequeñas empresas.

- El atractivo de la informalidad como opción de flexibilidad e independencia ante la percepción de escasos beneficios de la formalidad.

- La reducida fiscalización y la escasa sanción social. (OIT, 2015, p. 13)

Las dinámicas de formalización que se proponen en este proyecto se deben aplicar a unos tipos de negocios muy específicos, los pequeños negocios ubicados en la Comuna 10 de la ciudad de Medellín. Ahora bien, cabe preguntarse: ¿Qué son los pequeños negocios o negocios barriales? 
Para Páramo (2015) el negocio barrial, que denomina Mype, se pueden definir como el que no pasa de los diez empleados y por dinámicas de mercado o por decisión de vida se enfrenta a diversas limitaciones desde el punto de vista comercial, logístico y financiero. El negocio barrial, además de convertirse en una institución económica regida por el mercado, también es una institución social por el conjunto de relaciones que se tejen entre el empresario y la comunidad.

Uno de los negocios más tradicionales de este tipo son las tiendas de barrio. De acuerdo con Tapias, este tipo de negocios son

generalmente carentes de tecnología, [...] abastecen a las personas que por sus ingresos y localización no pueden aprovechar las ventajas económicas de comprar semanal o quincenalmente, y [...] adicionalmente proveen de otros servicios [...] como [...] otorgar crédito y horarios más amplios. $(1997$, p. 1)

El estudio de Páramo (2015) identifica algunas de las características más relevantes de los negocios barriales: el crecimiento no es su mayor preocupación, se legitiman de acuerdo a las condiciones de modelo social en el cual operan, muchos de ellos son familiares y tienen menos de diez empleados, las actividades se concentran en los propietarios como eje del negocio, no existe planeación de largo plazo y se toman decisiones inmediatistas, no hay vigilancia estratégica de mercados, la proyección comercial se desarrolla a partir de potenciar las capacidades y las relaciones comerciales se basan en la construcción de relaciones de confianza.

Adicionalmente, los resultados del estudio de Londoño (2016) sobre caracterización de los negocios barriales del borde oriental de la Comuna 10 de la ciudad de Medellín permiten identificar algunas características de funcionamiento de estos pequeńos negocios. Entre las más importantes están: la estructura comercial es pequeña, el mayor canal de contacto con el cliente es la presencialidad, bajo nivel de bancarización debido a los flujos de recursos y la desconfianza en las entidades financieras, altos niveles de informalidad en la estructura contable de los negocios, si bien se cumplen requisitos no existe una cultura de la formalidad en la gestión, la capacidad de generar empleo es baja y no hay un acceso integral a la seguridad social para los colaboradores del negocio. 


\section{La historia de los negocios barriales en Colombia}

A finales del siglo XIX los barrios en Colombia apenas y se asemejaban a asentamientos humanos urbanos. Es a mediados del siglo XX que la dinámica y la fuerza social de las comunidades conforman los barrios, en donde se desarrolla y concentra la cotidianidad de los individuos.

En los barrios de las grandes ciudades colombianas, y alimentadas por los procesos de migración generados por la violencia en los campos, se empezaron a desarrollar actividades económicas orientadas a generar ingresos de subsistencia. Muchas familias empiezan en las salas o garajes a vender productos de la canasta familiar, "tintos", empanadas, papitas fritas, arepas, carne asada, entre otros comestibles, y a ofrecer servicios de arreglos de modistería, plomería y reparaciones eléctricas.

En conclusión, se empieza a configurar un conjunto de economías locales que con el paso del tiempo se van expandiendo y van más allá de establecer relaciones económicas para articular relaciones sociales. Estos negocios hacen parte de la cultura y el tejido social de los barrios.

Así, gran cantidad de negocios se van configurando bajo parámetros de alta informalidad. La OIT (2015) identifica tres elementos relacionados en ese patrón: el sector informal, el empleo informal y la economía informal. Estas tres dinámicas se pueden observar en la economía barrial. En primer lugar, las actividades económicas desarrolladas no demandan altos niveles de especialización; es muy sencillo montar un negocio en el contexto de una economía local, por lo cual estos van surgiendo de manera espontánea sin cumplir con ningún requisito de formalidad. En segundo lugar, los negocios de barrio se constituyen como negocios familiares, la misma familia es la que trabaja allí.

Desde inicios de la década anterior el gobierno viene incorporando los procesos de formalización empresarial como objetivos fundamentales de la política económica. Entre las opciones definidas por las autoridades económicas se encuentran la simplificación de los trámites para crear empresa y la reducción de costos para operar bajo criterios de formalidad. 
Las medidas de formalización empresarial en Colombia inician con la Ley 590 de 2000 o "Ley Mipyme". Dentro de las medidas más importantes definidas en esta ley se cuentan las condiciones para el microcrédito empresarial bajo las reglas del juego del sistema financiero formal y los beneficios tributarios para la creación de nuevas empresas y la formalización de las existentes.

Posteriormente, en la Ley 905 de 2004 se proponen algunas medidas adicionales para el fomento de la mipyme. Entre ellas se cuentan el acceso preferencial a los mercados de bienes y servicios del Estado, la creación del Fondo de Promoción y Desarrollo Tecnológico de las Mipymes y el trabajo articulado con las entidades responsables de los procesos de formalización locales. En el caso que nos ocupa la responsabilidad por la formalización recae en la Alcaldía y la Cámara de Comercio de la ciudad de Medellín.

En la reforma tributaria de 2016 el Gobierno dio a las empresas cuyas ventas fueran inferiores a los cien millones anuales la alternativa de un impuesto denominado "monotributo", que simplifica en una sola las contribuciones de IVA, renta y pagos de seguridad social. Este impuesto trae dentro de sus ventajas la inclusión financiera. Respecto de su aplicación, el diario Portafolio (2016) presenta el siguiente ejemplo:

Con la creación de esta figura, básicamente los pequeños comerciantes con ventas entre 41 y 100 millones de pesos al ańo pagarían entre 40000 y 80000 pesos mensuales con acceso al ahorro para la vejez (Beps) y riesgos profesionales, y cubriría a los comerciantes ubicados en locales de menos de 50 metros cuadrados.

\section{El contexto de los negocios barriales en Colombia}

La estructura empresarial colombiana tiene sus bases en las micro, pequeñas y medianas empresas, que son consideradas el eje fundamental del dinamismo económico, político y social. Las mipymes son capaces de generar altos índices de empleo, frenar la concentración de los grandes capitales, diversificar el mercado nacional 
e internacional, fortalecer la cadena productiva, innovar, estimular y garantizar el continuo desarrollo empresarial. A continuación se presenta la clasificación de las pymes en Colombia, así como sus obligaciones tributarias (tablas 1 y 2).

Tabla 1. Clasificación de las pymes en Colombia

\begin{tabular}{lcr}
\multicolumn{1}{c}{ Clasificación } & Personal vinculado & Activos totales \\
\hline Mediana empresa & $51-200$ & $5001-15000$ SMLMV \\
Pequeña empresa & $11-50$ & $501-5001$ SMLMV \\
Microempresa & $1-10$ & Menos de 501 SMLMV
\end{tabular}

Fuente: elaboración propia con base en Ley 905 de 2004.

Tabla 2. Clasificación de empresas según obligaciones tributarias

$\begin{array}{ll}\text { Las pequeñas } & \begin{array}{l}\text { Microempresas, cuyo nivel máximo de ventas se ubica por debajo del } \\ \text { umbral tributario. Hacen una declaración fiel y no pagan impuestos. Se } \\ \text { conocen como declarantes no contribuyentes }\end{array} \\ \text { Las que ajustan } & \begin{array}{l}\text { Son un poco más grandes y su nivel máximo de ventas se sitúa por encima } \\ \text { del umbral, pero deciden, de manera legal, operar apenas por debajo del } \\ \text { umbral tributario a fin de no pagar impuestos ni costos de cumplimiento }\end{array} \\ \text { Las fantasmas } & \begin{array}{l}\text { Empresas cuyo nivel real de ventas se sitúa por encima del umbral } \\ \text { tributario, pero que deciden declarar, de manera fraudulenta, que se } \\ \text { sitúan por debajo del umbral tributario o no declarar nada en absoluto }\end{array} \\ \text { Las que estafan } & \begin{array}{l}\text { Producen por encima del umbral y declaran tan solo una parte de sus } \\ \text { ventas }\end{array} \\ \text { Las grandes } & \text { Hacen una declaración fiel y pagan el monto total de los impuestos }\end{array}$

Fuente: Kanbur y Keen, 2014. 
La mayor parte de la estructura empresarial barrial está conformada por un conglomerado de micro y pequeñas empresas que configuran las lógicas de una forma de relación económica y social en la que interactúan vecinos en espacios territoriales muy cerrados denominados barrios. Después de presentar la clasificación empresarial (tabla 2) nos ocuparemos de algunas de esas lógicas de la economía barrial, y específicamente del funcionamiento de estas realidades en la Comuna 10 de Medellín.

La economía barrial es un concepto que se ha venido consolidando con el paso del tiempo en los escenarios de las economías locales. Al respecto, el estudio de Arango y Arbeláez (2015) identifica los sectores más representativos de la economía popular analizando las realidades empresariales en el centro de la ciudad.

Los resultados dieron cuenta de los siguientes sectores: vendedores callejeros; graneros, tiendas y misceláneas; peluquerías, piñaterías, variedades, juguetería y papelerías; talleres de reparación de diversos artículos; natilleras; panaderías; tramitadores; comidas rápidas tradicionales; talleres de barrio; confecciones y maquila textil; transporte informal; minutos a celular; cafés internet y famihoteles.

Ese conglomerado de pequeños negocios de todo tipo que encontramos en los barrios, sujeto a unas relaciones económicas, políticas y sociales muy diferentes a las de las grandes empresas, se constituye en un mundo lleno de realidades complejas, así como de oportunidades para la sociedad, la academia y muchos de los entes encargados de la toma de decisiones de política pública tendientes a fortalecer el desarrollo empresarial.

Una de esas realidades complejas que a la vez se convierte en un reto para la política de desarrollo empresarial es la informalidad. De acuerdo con los criterios definidos por la OIT en 2015, tal concepto hace referencia a un

conjunto de empresas no registradas o no jurídicamente constituidas en la economía. Son empresas no registradas cuando no se han registrado bajo las formas específicas que exige la legislación vigente en el país, ya sea en el ámbito comercial, fiscal, laboral o administrativo. (Deelen, 2015, p. 26) 
Todas estas dinámicas de informalidad y toda la aplicación de los programas de formalización empresarial liderados desde la OIT, que en el caso de Medellín son liderados por la Administración Municipal, se desarrollan en un espacio geográfico definido: la Comuna 10.

Según los datos de la encuesta de calidad de vida de 2016 en la Comuna 10, La Candelaria, se contabilizan 85587 habitantes fijos. Pero día a día cerca de dos millones de personas están visitando los diferentes sectores del centro de la ciudad, lo cual determina la existencia de una importante dinámica económica materializada en diferentes tipos de negocios y de relaciones económicas, muchas de las cuales se desarrollan bajo criterios de informalidad.

La encuesta de calidad de vida de Medellín para 2016 resalta lo siguiente con respecto a la estructura empresarial de la ciudad:

Los sectores con mayor crecimiento neto en el número de empresas fueron los de seguros y finanzas, y comercio, restaurantes y hoteles. La estructura empresarial de la ciudad permaneció estable en relación con los años anteriores, con la mayor proporción de la base empresarial ocupada por microempresas, seguidas de pequeñas, medianas y grandes empresas.

Por comunas, el mayor número de empresas sigue teniéndolo La Candelaria, seguida de El Poblado y Laureles-Estadio. La Candelaria concentra una mayor proporción de empresas en sectores con alta informalidad laboral, mientras que El Poblado tiene una mayor participación de empresas con un nivel de formalidad relativamente alto, de acuerdo con cifras para las ciudades colombianas del Dane. (Medellín Cómo Vamos, 2017, p. 180)

De manera más específica, los datos de la estructura empresarial de la Cámara de Comercio para 2017 dan cuenta de la existencia de 20735 negocios en La Candelaria (Comuna 10). La figura 1 representa las actividades económicas con mayor concentración de negocios. 
Figura 1. Estructura empresarial Comuna 10 de Medellín por actividad económica

Industrias manufactureras

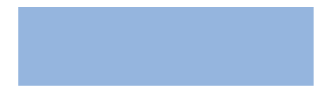
3327

Comercio al por mayor y al por menor; reparación de vehículos automotores y motocicletas

Alojamiento y servicios de comida 1946

Actividades profesionales, científicas y técnicas

Fuente: elaboración propia con datos de Cámara de Comercio de Medellín, 2018.

En cuanto a las participaciones porcentuales, el $46.62 \%$ de los negocios están dedicados a actividades de comercio al por mayor y al por menor; el segundo renglón económico más importante es el de las actividades manufactureras, con el 16.04 \% de los negocios, y en tercer lugar están los alojamientos y servicios de comida, que representan el $9.38 \%$ del tejido empresarial de la comuna.

Los resultados de la figura 2 evidencian que el $88.29 \%$ de los negocios de la Comuna 10 son microempresas: 18309 negocios que tienen menos de 10 empleados y cuentan con activos inferiores a $\$ 368858500$, umbral que para muchos de estos negocios es incluso una utopía.

Un análisis integrado de las figuras 1 y 2 da cuenta de una estructura empresarial compuesta en su gran mayoría por pequeños negocios concentrados en cuatro actividades económicas principales: comercio al por mayor y al por menor, industrias manufactureras, servicios de alojamiento y restaurante, y actividades profesionales, científicas y técnicas. 
Figura 2. Estructura empresarial Comuna 10 de Medellín por tamaño de negocios

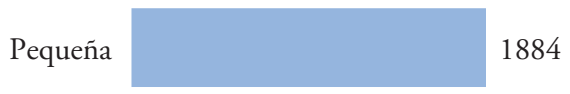

Mediana

Grande 96

Fuente: elaboración propia con datos de la estructura empresarial de la Cámara de Comercio de Medellín, 2018.

Complementando el análisis anterior, la encuesta de calidad de vida de 2016 para la ciudad de Medellín plantea lo siguiente: "En 2016 las pequeñas empresas del sector de comercio, restaurantes y hoteles, transporte, almacenamiento y comunicaciones, y construcción, son las que tienen mayores niveles de informalidad en las principales ciudades colombianas" (Medellín Cómo Vamos, 2017, p. 188). En ese sentido, se debe recordar que el censo empresarial resaltaba que en la comuna existían 9668 unidades económicas dedicadas a actividades de comercio al por mayor y al por menor, así que la cita anterior es indicio de que muchas de estas empresas funcionan bajo condiciones de informalidad.

Además, el estudio de políticas de formalización de pymes en América Latina realizado por la OIT planteaba que las mipymes (micro y pequeñas empresas) eran más propensas a la informalidad por cuatro razones básicas: "Baja productividad y altos costos de ser formal; complejidad de procedimientos, regulación inadecuada para las características de las micro y pequeñas empresas; informalidad como opción atractiva de flexibilidad e independencia; y reducido control y fiscalización, escasa sanción social" (Deelen, 2015, p. 41). 
En este orden de ideas, y en coherencia con las políticas locales y nacionales que buscan la formalización, este proyecto de investigación pretende abordar la incidencia de los procesos de formalización contable y tributaria en el desempeño empresarial de los negocios barriales ubicados en la Comuna 10 de la ciudad de Medellín. Además, espera dar cuenta, como lo prevé el profesor Cadena (2018), de que cada vez se torna más necesario actualizar el Código Civil colombiano en razón a que las reformas y contextualizaciones que se le han hecho a la normativa son coyunturales, parciales y desarticuladas frente a las realidades sociales e institucionales del país, que por cierto ha tenido profundas transformaciones en los últimos años.

\section{Conclusiones}

La estructura empresarial revisada carece de una contabilidad estructurada, puesto que la mayoría de organizaciones son pequeńos negocios concentrados en cuatro actividades económicas principales: comercio al por mayor y al detal, industrias manufactureras, servicios de alojamiento y restaurante, y actividades profesionales, científicas y técnicas. Tales actividades, por su objeto social, la cantidad de activos que manejan y los ingresos que perciben no requieren de un sistema contable que refleje la realidad de las organizaciones.

Conforme con lo planteado por la OIT en su estudio para América Latina, las condiciones que más propician la carencia de herramientas contables, financieras y tributarias por la población objeto de investigación son la baja productividad y los altos costos de ser formal; la complejidad de procedimientos y la regulación inadecuada para las características de las micro y pequeńas empresas. La informalidad en las micro y pequeñas empresas se constituye en un elemento fundamental para la baja adopción de sistemas contables y tributarios, lo que aumenta el riesgo de quiebra de este tipo de organizaciones, puesto que al no conocerse su realidad financiera no se pueden tomar decisiones específicas en términos de proveedores, sistemas de costos e incluso elementos emergentes de la contabilidad, como los costos ambientales y sociales. Todo eso es ocasionado por la cultura de la informalidad, las altas tasas impositivas para los formalizados y la adaptación de la comunidad a este tipo de organizaciones. 


\section{REFERENCIAS}

Arango, M. y Arbeláez, J. (2015). La economía popular: alternativa empresarial exitosa. Medellín: Fondo Editorial María Cano.

Cadena, W. (2018). El hermano hombre de Fernando Soto Aparicio. Via Inveniendi et Iudicandi, 13(1), 237-262. Doi: http://dx.doi.org/10.15332/s1909-0528.2018.0001.09

Cámara de Comercio de Medellín para Antioquia (2018). Estructura empresarial 2017. Recuperado de http://www.camaramedellin.com.co/site/Servicios-Empresariales/Informacion-Empresarial/Estadisticas-Camara.aspx

Castro, G. (2018a). Una aproximación teórica a la obra de Arturo Valencia Zea De la posesión y la función social de la propiedad: el gran problema jurídico del siglo XX en Colombia, revisión histórico jurídica. Via Inveniendi et Iudicandi, 13(1), 9-54. Doi: http:// dx.doi.org/10.15332/ s1909-0528.2018.0001.01

Castro, G. (2018b). Una nueva propiedad intelectual para el siglo XXI. Focos, críticas y propuestas puntuales para una futura regulación de los bienes inmateriales. Revista Iusta, 1(48), 19-41. Doi: https://doi.org/10.15332/s1900-0448.2018.0048.01

Deelen, L. (ed.) (2015). Politicas para la formalización de las micro y pequeñas empresas en América Latina. Santiago de Chile: OIT. Recuperado de https://goo.gl/QHJsvK

Dinero (2016). El $21 \%$ de los negocios en Colombia son tiendas de barrio. Recuperado de https://www.dinero.com/economia/articulo/tiendas-de-barrio-y-negocios-mascomunes-en-colombia/224631

Herrera, B. (2018), Derechos de los animales: la legislación nacional interna como barrera legal para el reconocimiento de la subjetividad jurídica animal. Via Inveniendi et Iudicandi, 13(1), 55-93. Doi: http://dx.doi. org/10.15332/s1909-0528.2018.0001.02

Hurtado, M. (2017). Crisis de la forma jurídica y el despertar antisistémico: una mirada desde el pluralismo jurídico de las Juntas de Buen Gobierno (JBG). Revista Iusta, 2(47), 19-33. Doi: http://dx.doi.org/10.15332/s1900-0448.2017.0047.01 
Kanbur, R. y Keen, M. (marzo de 2015). Reducir la informalidad. Finanzas \& Desarrollo. Recuperado de https://goo.gl/oPWUur

Llano, J. (2017). Teoría del derecho, neoconstitucionalismo y derechos diferenciados. Verba Iuris, 12(38), 13-32.

Londoño, C. (2016). Caracterización de los negocios barriales del borde oriental de la Comuna 10 de la ciudad de Medellín. Medellín: Fundación Universitaria María Cano.

Medellín Cómo Vamos (2017). Informe de indicadores objetivos sobre la calidad de vida en Medellin, 2016. Recuperado de https://goo.gl/whNNt4

OIT (Organización Internacional del Trabajo) (2014). Políticas para la formalización de las micro y pequeñas empresas en Colombia. Recuperado de https://goo.gl/RL6vBo

Páramo, D. (2015). Presente y futuro de los negocios de barrio (Mypes). Barranquilla: Escuela de Negocios, Universidad del Norte.

Portafolio (2016). El monotributo no asusta a los comerciantes, pero quieren tiempo para analizarlo. Recuperado de https://goo.gl/5XyuNT

Senado de la República de Colombia (2000). Ley 590. Recuperado de https://goo.gl/ ZNaFNG

Senado de la República de Colombia (2004). Ley 905. Recuperado de https://goo.gl/ GuDoQ4

Tapias, C. (1997). La gran tienda de barrio. Sotavento MBA, 6, 60-71.

Torres, Y. y Ramírez, O. (2018). La responsabilidad civil de las entidades de certificación en Colombia. Revista Iusta, 1(48), 43-71. Doi: https://doi.org/10.15332/ s1900-0448.2018.0048.02 
